\title{
Perspectives on Animal Models Utilized for the Research and Development of Regenerative Therapies for Articular Cartilage
}

\author{
Dan Xing ${ }^{1}$ Jiaqing Chen ${ }^{2}$ J Jiabei Yang ${ }^{2}$ Boon Chin Heng ${ }^{3}$. \\ Zigang $\mathrm{Ge}^{2,4}$. Jianhao Lin $^{1}$
}

Published online: 5 May 2016

(C) Springer International Publishing AG 2016

\begin{abstract}
Animal models are integral and indispensable for biomedical research and regenerative medicine studies, as these provide invaluable information for systemically evaluating the potential risks and efficacy of newly developed biomaterials, drugs, medical devices, and therapeutic modalities, prior to initiation of human clinical trials. Nevertheless, it is important to be aware of the unique strengths and limitations of the various different small and large animal models commonly utilized for biomedical research as well as the various challenges faced in extrapolating results acquired from animal studies and the risks of data misinterpretation. This review will thus critically examine various animal models utilized for studies on articular cartilage regeneration. Particular emphasis will be placed on comparing and analyzing the unique strengths and limitations of each animal model, with the aim of establishing principles for evaluating the suitability of
\end{abstract}

This article is part of the Topical Collection on Preclinical Animal Models in Regenerative Medicine

Zigang Ge

gez@pku.edu.cn

$\triangle$ Jianhao Lin

linjianhao@pkuph.edu.cn

$1 \quad$ Arthritis Clinic and Research Center, Peking University People's Hospital, Beijing 100044, China

2 Department of Biomedical Engineering, College of Engineering, Peking University, Beijing 100871, China

3 Faculty of Dentistry, The University of Hong Kong, Pokfulam, Hong Kong

4 China Orthopaedic Regenerative Medicine Group, Hangzhou, Peoples Republic of China different animal models for individual studies as well as for comprehensive interpretation and extrapolation of results obtained from various animal species. Additionally, this review will also discuss to evaluate animal studies with in situ imaging techniques, how the animal genome may result in variability in experimental outcomes, as well as the contribution of animal models to the development of cartilage tissue engineering.

Keywords Animal model · Articular cartilage . Regeneration $\cdot$ Microfracture $\cdot$ Systematic review

\section{Introduction}

It is often challenging to emulate in vivo studies with highthroughput and standardized in vitro screening assays in biomedical research, which makes the use of animal models indispensable in most cases. However, the lack of comprehensive understanding of animal models limits our ability to extrapolate research data acquired from animal studies to human clinical practices, which in turn results in misinterpretation of data and unnecessary wastage of experimental animals [1]. Non-human primates may serve as an alternative option. Nevertheless, in practical terms, it is often necessary to balance multiple aspects of the cost-benefit axis, such as in vitro versus in vivo, small animals (rat, rabbit, and dog) versus large animals (sheep, goat, pig, and horse), scientific gain versus animal welfare, as well as value for money [2]. From innumerable ideas and prototypes, to millions of small animals and hundreds of thousands of large animals utilized, to thousands of animal studies and clinical trials being initiated per year (from in vitro models to small animals, large animals, primates, and human beings) (estimated from https:// clinicaltrials.gov/), this disparity poses significant challenges 
and raises many pertinent questions: i.e., "Have we utilized animal studies wisely and efficiently?" "Have we extrapolated data from animal studies correctly?" "How can we compare and analyze data from varied and diverse animal species?" With the accumulation of research knowledge and data over the past few decades, we are now at an even stronger position to tackle this challenge than ever.

Amongst the various different animal disease models utilized in preclinical research, we would like to focus on the articular cartilage defect model in this review. Articular cartilage possesses low intrinsic regenerative capacity [3], which could be a leading cause of why half of the world's population aged 65 years and above suffer from osteoarthritis (OA) [4]. Cartilage injury-induced OA is implicated in at least $12 \%$ of all OA cases [5]. However, the underlying mechanisms of cartilage regeneration are as yet not fully understood [6]. Cartilage regeneration is an extremely complicated biological process involving a diverse multitude of different mechanisms, such as inflammation, mechanical loading, recruitment of stem/progenitor cells, as well as clinical interventions [7].

The advantages and limitations of individual animal models in articular cartilage research have been extensively reviewed elsewhere [8]. Ahern et al. conducted a systematic review to critically examine the advantages and limitations of different preclinical animal models of cartilage defects [9]. However, further perspectives and comprehensive discussions are required. In particular, input-output ratio and animalhuman extrapolation need to be evaluated. Each individual animal model could provide some meaningful information [10]. Small animals (rabbits, rats, and mice) are usually utilized for the purpose of initial evaluation and biological compatibility tests, but these only provide limited information, as the joints of small animal tend to heal more readily and spontaneously than the human clinical model. Due to ethical considerations, large non-companion animals (sheep, goat, and pigs) are preferred to companion animals (dogs and horses) $[11 \bullet \bullet$. Moreover, new techniques such as minimally invasive methods and biomechanical tests can be applied to large animals. Despite having much similarity to humans, non-human primates have been seldom utilized in cartilage regeneration research, due to scarcity, high costs, ethical consideration, and high profile in animal welfare and also because these are often unable to provide additional information beyond the aforementioned large animal models [12].

The purpose of this review is to systematically and critically examine the different animal models utilized for investigating new regenerative therapies of articular cartilage, through evaluating selection of animal models, proportion of sacrificed animals, animal-clinical extrapolation, results justification, and comparison between various species and anatomical locations. A systematic search of PubMed-listed publications was used to identify relevant studies from October 2005 to October 2015 that were related to animal models and regeneration of articular cartilage. The medical subject headings (MeSH; National Library of Medicine, Bethesda, MD) "models," "animal," "cartilage," "articular," "regeneration," and "cartilage" and the free-text words "animal models" or "articular cartilage" were combined, as presented in Appendix Table 2. The contribution of animal models to cartilage tissue engineering was summarized according to the statistical data obtained from results of the systematic search.

\section{Animal Welfare, Ethical Approval, and Minimization of Numbers of Animal Utilized}

Necessity of animal studies has to be determined, before any animal study is designed and gets approval. Estimation of the sample size of experimental animals have to be kept minimal according to forms and variation of data, while experimental animals have to be well kept and maintained in accordance with international standards [13]. Firstly, all experimental studies utilizing animal models should be conducted according to the International Guiding Principles for Biomedical Research Involving Animals that emphasize the $3 \mathrm{R}$ principles (reduction, replacement, and refinement) [14] or Public Health Service Policy on Humane Care and Use of Laboratory Animals [15]. Secondly, in vivo studies based on live animals must get approval from the ethics committee of the relevant local institution. Thirdly, it is recommended that surgical implantation of a tissue engineering construct into any animal model should be performed according to standard protocols [16].

It is essential to minimize numbers of animal used as well as utilize less intelligent species, avoiding the usage of dog and non-human primates where possible. In general, using inbred strain animals can reduce the required numbers of individual animals because of uniformity in their genetic background. However, higher prices of inbred strain animals will limit their usage as surgical animal models. The number of animals required is usually determined based on several parameters, such as variation, species, and prior experience. Although small animals have priority to be selected under rational circumstances [17], large animals are often irreplaceable due to more similar biomechanical functions and physiological responses to human beings.

\section{Animal Models Used in Articular Cartilage Research}

As microfracture is the most widely used regenerative therapy for cartilage in current clinical practice, drilling varied holes through cartilages with an aim to recruit stem cells/progenitor cells from bone marrow is widely utilized in animal studies [18]. The microfracture procedure [19] involves debridement of the lesion to a stable rim to enable the lesion to be well contained. Subsequently, the calcified cartilage layer is 
removed with a curette, and the subchondral bone is then penetrated with microfracture awls utilizing minimal force to achieve 3 to 4 perforations $/ \mathrm{cm}^{2}$. In order to allow extrusion of fat and blood droplets containing stem cells and growth factors from the bone marrow, sufficient depth of penetration by the awl is required. At the base of the prepared chondral lesion, bone marrow-derived mesenchymal stem cells (BMSCs) and growth factors infiltrate into the fibrin clot and subsequently generate fibrocartilaginous repair tissue [20]. However, fibrocartilage lacks the biomechanical features of native cartilage and becomes fibrous, which results in wearing out of the newly repaired cartilage tissue under physiological loads [21]. On the other hand, modified procedures are often used in cartilage defect models, which involve drilling holes in articular cartilage into subchondral bone with varying depths [22]. Various strategies have been used to improve quality and quantity of regenerated cartilage [23].

\section{In Vitro Model}

Effective cartilage regeneration involves orchestration and coordination of external and internal signals spatiotemporally with partial mimicking of embryonic development. In vitro models can be used to investigate some factors, which is difficult to analyze in vivo. Novel 3D cell culture systems [24], biomaterial scaffolds [25], shear/compression bioreactors [26], stem cell differentiation techniques [27], and analytical procedures could recapitulate functional cartilage regeneration in vitro [28 ]. To model exogenous intervention, articular chondrocytes were cultured within a collagen sponge in the presence or absence of IL-1 $\beta$ to generate cartilage in vitro, which enabled researchers to study the responses of chondrocytes to inflammatory cytokines [29]. This in vitro model aimed to screen the effects of compounds with therapeutic potential in osteoarthritis. When chondrocytes transduced with different genes were cultured in vitro, improved cartilage regeneration would be expected to correlate with specific chondrocyte phenotypes [30,31].

On the other hand, in vitro models are often limited by lack of mechanical stimuli, maturation of regenerated cartilage tissue, and absence of inflammatory responses [28•]. It is one of the most critical problems that in vivo and in vitro pliability of multi-potent stem cells and chondrocytes largely depends on their microenvironment. Various signaling cues, cytokines, and growth factors from the cellular microenvironment are crucial for the differentiation, proliferation, and maintenance of differentiated stem cells [32]. Phenotypes of progenitor/ stem cells and chondrocytes can be altered by soluble factors from the surrounding tissue, by paracrine signals from neighboring cells or by direct cellular contact [33]. Although several co-culture systems provide valuable information regarding molecular control in vitro that aim to mimic the physiological conditions at the injured cartilage site [34,35], functional regeneration of cartilage with articular characteristics is still a challenge. As there is still a long way to go to comprehensively understand spatial-temporal regulation of in vivo cartilage regeneration, promising results from in vitro studies have to be further evaluated by in vivo studies.

\section{In Vivo Model}

As utility, total expenses, cost-benefit considerations, and aims of studies have to be considered in selection of animal models, it is often challenging to balance these various conflicting parameters (Table 1). Small animal models, such as murine, lapine, canine, and caprine, are broadly used. Chondrogenesis has been studied with subcutaneous [36] and intramuscular implantation in nude mice [37]. The reasonable costs of animal purchase and care together with ease of handling and caging are the advantages of mouse models. High reproductive capacity and short duration of the reproductive cycle make mice more commonly utilized in genetic studies. However, relatively small joints and the thinner cartilage of mice would limit the usefulness of cartilage defect models in murine models [38-40]. To date, nude mice as well as transgenic or gene knockout mice have been utilized for studies involving ectopic chondrogenesis [31, 38, 41]. Rats have bigger joints and thicker cartilage than mice [40, 42], which make them more easily utilized in cartilage defect models than mice $[43,44]$. In the meantime, transgenic rats have demonstrated some advantages as cartilage defect models $[45,46]$. Nevertheless, caution has to be exercised in attempting to extrapolate results from rats to both large animal and human clinical models, as rat cartilage generally has better and continuous healing potential, which would obviously exaggerate the results [42].

Rabbits are the most widely utilized animal model in cartilage regeneration research. Their relatively larger joint size and thicker cartilages, compared with rats and mice, would increase their usefulness significantly. Full- or half-thickness cartilage defect models could be constructed in rabbits [47-49], with the subchondral bone being involved in $90 \%$ of cases. The main limitation of rabbit models includes spontaneous healing potential, with rabbit cartilage possessing greater intrinsic healing capacity than larger animals such as horses and humans $[50,51]$. A $3 \mathrm{~mm}$ diameter has been considered the critical size of cartilage defect to prevent spontaneous healing [52]. The higher flexion in knee joints of rabbits makes it difficult to extrapolate results obtained from the rabbit model to humans [10].

Dogs are often utilized in cartilage regeneration research due to their advantages in having larger joints, thicker cartilage, low intrinsic healing capacity, and similar mechanical properties of cartilage to humans [42]. Dog models are also widely used in surgical research. The cartilage thickness allows for surgical creation of defects involving the articular 
cartilage without the subchondral bone. Moreover, dogs are easily trainable after implantation surgery according to rehabilitation protocol, making these animals appropriate for exercise and physiotherapy studies [53]. Arthroscopic evaluation of the knee joint is feasible in dog models instead of animal sacrifice [54]. However, usage of dog models is limited in cartilage regeneration research due to relatively small defect volumes compared with primates, in addition to ethical reasons pertaining to their companion animal status [9].

Pig models for partial- or full-thickness cartilage defects were also used in several studies [55]. Thicker cartilage (1.5-2.0 mm) [56], up-right (instead of squatting) knee, and larger joint sizes are advantages of the porcine cartilage defect model. Nevertheless, pigs are characterized by difficult handling, large size, requirements in housing, and aggressive demeanor, while mini-pigs could offset some of these shortcomings [55]. It is critical to use mature mini-pigs to diminish the influence of spontaneous cartilage repair, as immature minipig cartilage (less than 42-52 weeks) has a relatively high spontaneous healing capacity [57].

The ovine model also has certain advantages such as large joint size, thicker cartilage, and lower spontaneous repair capacity, but late maturity and variability of cartilage thickness limit their wide usage for in vivo studies $[58,59]$. The variable thickness $(0.4-1.0 \mathrm{~mm})$ of cartilage in sheep makes the defect volume different between individual animals. This could in turn confound the replicability of results of in vivo studies. Another disadvantage of the ovine model is the very dense and hard subchondral bone, which could restrict choices of study design for cartilage regeneration requiring bleeding of the subchondral bone bed.

Goats are not costly and easier to handle than other large animals. Cartilage thickness in goats allows for creation of partial and complete thickness defects. Similar with sheep, the high variability of goat cartilage thickness could lead to variations of the volume of cartilage and subchondral bone defects within studies. On the other hand, compared to the sheep model, the subchondral bone of goat is softer and more compatible with normal surgical techniques for creating osteochondral defects. Thus, the goat, being a large animal model, is useful for in vivo studies of cartilage defect regeneration [60-62].

Horses are the largest animal models used for studies of cartilage defect regeneration $[63,64]$. As an animal with a relatively long lifespan, a long-term model of cartilage injury can be created in the horse [64]. As the horse is an athletic animal, it is an ideal animal model for evaluating resurfacing technologies in chronic defects [9]. The most significant advantage is that the cartilage thickness (1.75-2.00) in the horse is similar to that in humans [56]. However, persistent standing position during rest will place sustained weight load on the knee joints, which will influence the in vivo results compared with the human body. The higher costs, difficulty of handling 
and caging, and physiological condition may also restrict its use in preclinical studies. Their weight and special biomechanical conditions in the knee joint will also affect extrapolation to cartilage defect regeneration in the human clinical model.

\section{How to Validate Results Acquired from Different Animal Models?}

Animal models are essential for the development of novel clinical therapeutic modalities. However, the utilization of individual animal model is limited by their unique pros and cons. Hence, we will systemically dissect and compare some key factors involved with an aim to comprehensively understand animal models. Particular attention will be focused on the creation of cartilage defects by drilling holes of $2.5-4 \mathrm{~mm}$ diameter within articular cartilage (Fig. 1).

\section{Anatomy}

Mice are extremely useful due to the diverse array of inbred and genetically modified mouse strains available. In recent

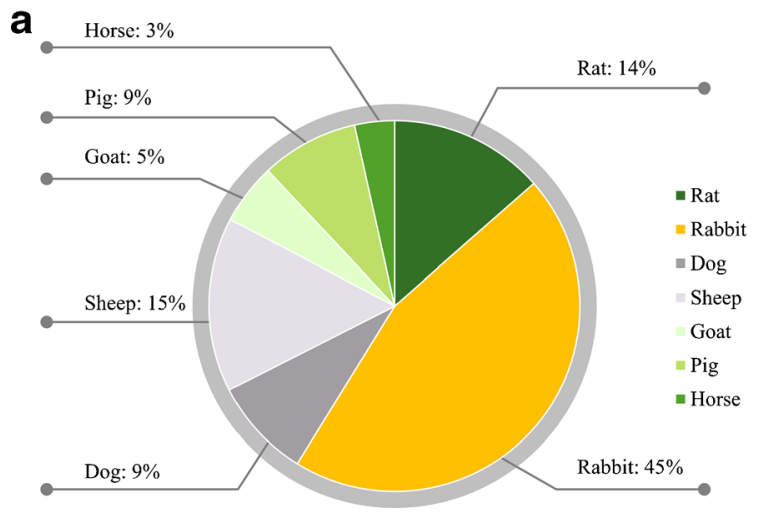

\section{C}

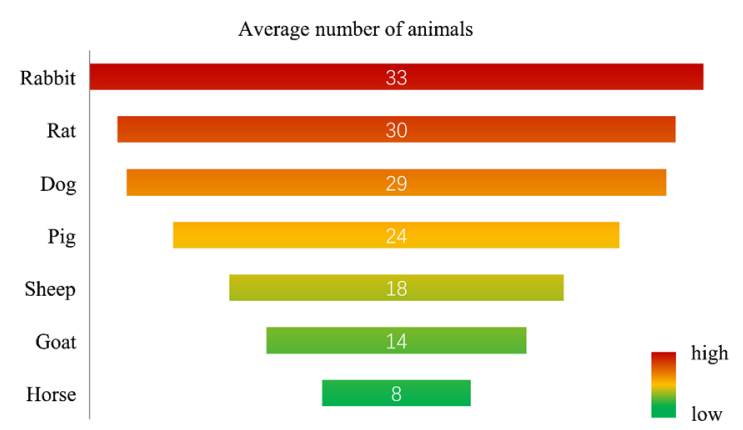

Fig. 1 Estimation of the proportion of various animal models utilized in cartilage tissue engineering research and relative contribution to in vivo studies before clinical application in the past 10 years. a The proportion of studies involving different animal models. Most studies utilized rabbit as an animal model in cartilage tissue engineering. b The proportion of animals sacrificed in the studies. More than half of the number of sacrificed animals comprises of rabbits. c Average sample size of each years, a number of mouse OA models have been described, including interior cruciate ligament rupture in the knee joint, cyclic tibial compression loading of articular cartilage, and intra-articular fracture of tibial subchondral bone [65]. Furthermore, multi-potential stem cells with or without scaffolds were usually implanted at the back of nude mice. However, murine cartilage defect models are not commonly utilized due to the smaller knee joints of mice.

Rodents are the most often utilized animal models to provide proof-of-concept data at the very beginning stages of research and development. The medial or lateral femoral condyle of rats could be used in full-thickness defect models involving the subchondral bone, but not as partial cartilage defect models due to its thinner cartilage. Studies involving ectopic chondrogenesis were often carried out at the back of nude mice [66, 67]. However, the lack of several key factors, such as similarity in biochemical characteristics, mechanical environment, and tissues interfaces, thus limits the value of results from rodent models. The lack of immunogenicity further limits the value of the nude mice model. The knee joints of rodents with a higher flexion degree have a different mechanical environment compared with the more erect position of humans.

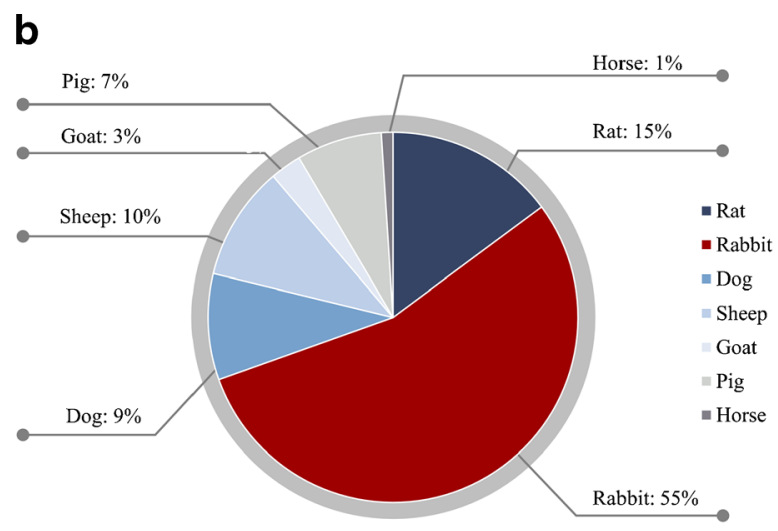

d

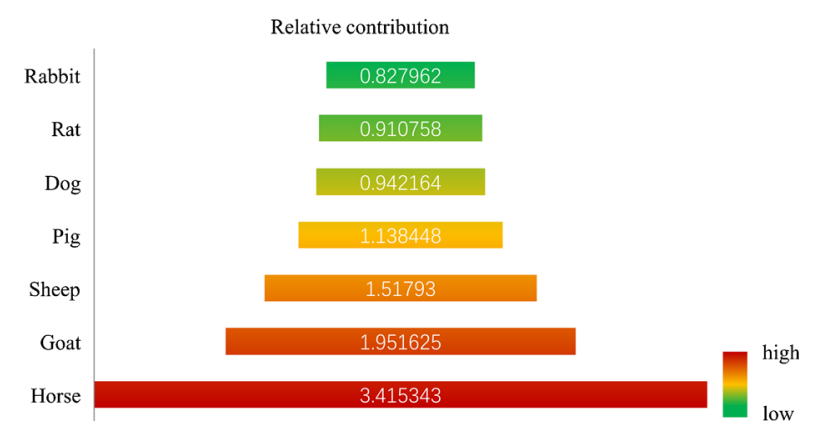

type of animal model. The trend of the average number of animals utilized in individual studies decreasing with increasing body weight is significant. d Relative contribution of each type of animal model in cartilage tissue engineering research. The trend of improving contribution of each type of animal model with increasing body weight is significant 
In large animals, partial- or full-thickness cartilage defects are often made in medial, lateral, or both femoral condyles. As it is difficult to construct partial-thickness cartilage defect models, most of the cartilage defect models in rabbit involve the subchondral bone, due to the variability of thickness of rabbit cartilage [68]. Anatomical selection includes the femoral trochlea and the medial or lateral femoral condyle in rabbit models [22].

Dogs are better models for cartilage defects, as the thicker cartilage thickness of dogs allows creation of full or partial defects of articular cartilage without involvement of subchondral bone [9, 69]. A majority of studies still utilize the osteochondral defect model in dogs [70,71]. Dog cartilage defects were selectively created in the femoral trochlea, the medial femoral condyle, and both condyles.

Sheep have articular cartilage of variable thickness, which leads to difficulty in comparing parameters amongst experimental groups. The particularly hard subchondral bone of sheep limits the choices of experiments that can be performed. In goat models, the subchondral bone is softer compared to sheep. Furthermore, the consistent thickness of goat cartilage allows for defect modeling in articular cartilage that more closely resembles human cartilage [72]. Defect location in sheep and goat includes the femoral trochlea, the medial femoral condyle, and both condyles.

Although pig is not regularly used as a cartilage defect model, adult mini-pig is occasionally utilized for studies involving the following anatomical locations: the femoral trochlea, the medial femoral condyle, and both condyles. The thickness of the cartilage of mini-pigs would meet the requirements for construction of partial- or full-thickness cartilage defect models [73].

$\mathrm{OA}$ is the leading cause of retirement of equine athletes. Special attention was given to the horse model recently [74]. Although the horse has equivalent joint size, the lateral femoral trochlea is the most common location for cartilage defects [75]. Cartilage defects of more than $350 \mathrm{~mm}^{2}$ can be produced without subchondral bone involvement. Defects have also been created in the lateral condyle of the metacarpophalangeal joint and the middle carpal bones. However, we should take their body weight and static loading into consideration when extrapolating the in vivo results.

\section{Age and Gender: How to Justify and Compare?}

It is generally agreed that young and adolescent animals have stronger capacity for cartilage regeneration, compared with older animals [76]. Hence, defect models in animals usually utilize mature adult animals to minimize spontaneous cartilage healing. Intrinsic healing abilities of the animals have to be seriously considered when investigating cartilage regeneration. For dogs, it is difficult to obtain consistent skeletally mature animals. This is probably because the age of skeletal maturity ranges from 12 to 24 months [77•]. Sheep and goats exhibit similar skeletal maturity at $2-3$ years of age [9]. The FDA states that mini-pigs reach skeletal maturity by 42 52 weeks. It is suggested that further studies should be conducted when skeletal maturity is reached. In practice, horses, retiring from various athletic careers, are often used for animal models but often require examination for coexistent joint diseases [9]. The skeletal maturity and timing of modeling are presented in Table 1.

\section{Follow-up Duration}

Currently, 3 and 6 months are the most frequently used time points after surgery. Twelve and 24 months are sometimes used to more rigorously evaluate potential degeneration of de novo cartilage, which actually happens quite often [6]. Nevertheless, the very beginning stages, say hours or days, are critical but often neglected, limited by current technology constraints. In practice, body weight and age are used as rough estimates. Intervals between sample harvests are often chosen according to experiences of individual researchers. The purpose of taking time points is to evaluate the healing progress dynamically, usually from the beginning stage to ultimate regeneration. In principle, cartilage regeneration in large animal models need longer duration compared to small animal species. Because large animals have a longer life span and relatively slow regeneration processes, the follow-up duration ranges from 4 to 24 weeks for ectopic chondrogenesis in nude mice models to between 2 and 78 weeks of follow-ups in rabbit, dog, and sheep cartilage defect models, while up to 104 weeks of follow-ups have been reported for the goat model and 1-52 weeks have been reported for the pig and horse models [9].

\section{Limitations of In Vivo Evaluation}

When interpreting results from in vivo studies, it is important to note possible bias in the presentation of histological and biochemical data, i.e., (i) slides with "optimal regeneration" have better chances to be presented, which are not representative of the entire tissue, (ii) the in vivo results which are consistent with that in vitro may get more attention than data that are inconsistent with the in vitro results, and (iii) the in vivo histological results may exhibit variability due to different morphologies. The best results of histological evaluation are often chosen for presentation or publication by investigators, a practice commonly referred to as "cherry-picking." Accordingly, while the results of previous individual studies should be considered in designing new experimental studies, these biases in subjective evaluation should be considered when interpreting the findings. 


\section{Perspectives}

\section{Integration of In Situ Imaging Technology with Animal Studies}

Currently, non-invasive evaluation technologies have provided innovative detection tools for in vivo studies. By using realtime and non-invasive techniques, the evaluation of live tissues in vivo could permit a better understanding of in situ regeneration dynamically. In vivo non-invasive evaluation technologies include functional mechanical testing (assessing the tensile, shear, and compressive properties of engineered cartilage), imaging technologies, and cell and growth factor tracking in animal models [78]. Indentation testing is a compressive test that offers a new method for in situ, nondestructive mechanical analysis of cartilage, which aims to quantify some biomechanical characteristics [79]. Magnetic resonance imaging (MRI) has been broadly used in clinical diagnostics of joint diseases but is limited by inconvenient access of experimental animals to facilities. The roughness of cartilage surface could be evaluated by ultrasound [80]. Non-invasive imaging of cartilage at the micrometer-level resolution based on detecting the equilibrium partitioning of an ionic contrast agent via microcomputed tomography enables in situ imaging of cartilage and bone simultaneously in three dimensions [81]. Fluorescent labeling has been used to track seeded cells [82], while superparamagnetic iron oxide (SPIO) magnetic nanoparticles have been used to label seeded cells in combination with MRI [83].

These technologies offer real-time and dynamic evaluation. However, several limitations of these technologies restrict their widespread utilization in animal models. These include the lack of appropriate resolution for large animals, the larger stature of some animals that is not suitable for some measurements, the potential harm from radiation exposure and contrast agents, and penetration into hard tissues.

\section{More Functional Parameters}

Information on matrix maturation is largely neglected or limited [84]. The intricate structure of articular cartilage imparts it with essential functions, including lubrication, load bearing, and transfer. The components, concentration, structure, and morphology are highly anisotropic in native mature articular cartilage. However, engineered cartilage can be immature with incomplete components and isotropic structure. The majority of studies evaluate cartilage formation by histology (e.g., type I/II/X collagen and glycosaminoglycan), immunohistochemistry, and gene expression, which are inadequate. Moreover, zonal structural data are usually omitted. Very often, there is a lack of further testing for better evaluation of engineered cartilage. It is necessary for us to improve on current therapeutic modalities to enable engineered cartilage tissue to be more similar to native cartilage. Hence, we need to pay more attention to components and structural features of cartilage regeneration, particularly anisotropic structural information. The extent of cartilage maturation can be roughly determined by tensile properties [85]. To be more precise, the zonal structure of engineered cartilage can be characterized by the picrosirius polarization method [86], for example. Fiber optic confocal imaging technology for 3D histology has been developed to supplement traditional histology and constructed confocal arthroscopy for in vivo imaging of chondrocytes in articular cartilage [87]. Mechanical testing, which is also important for better evaluation, is often lacking in many studies.

Although various methods can be selected, a comprehensive and standardized evaluation system for engineered cartilage can facilitate progress in the field. However, it is challenging to select key parameters. Furthermore, regenerated cartilage with zonal structure is still an enigma in the current field. Paying more attention to maturation of engineered cartilage can lead to better evaluation of cartilage regeneration as well as provide the basis for exploring further mechanisms.

\section{Clinical Applications}

Although several clinical studies on therapy of human cartilage defects have been conducted in the human body [88-90], the current evidence would suggest limited efficacy of most therapeutic modalities [91]. The reasons may include deficiencies in study designs, follow-up methods and duration, inclusion/exclusion criteria, and evaluation methods amongst these various studies. Despite the advancement of cartilage tissue engineering with in vitro or in vivo animal studies, human models or clinical trials are still required.

\section{Immunology: Integral to Cartilage Regeneration but Often Neglected}

The immune system is crucial in determining the quality of cartilage repair. It has been the consensus opinion that immunological factors are integral to tissue regeneration. However, its role in cartilage regeneration has not been fully characterized. Studies in diverse animal species have demonstrated an association between the loss of regenerative capacity and maturation of immune competence. However, other studies reported that the immune response enhances repair and ensures local tissue protection. Therefore, the role of the immune response in cartilage repair is rather complex. Additionally, mesenchymal stem cells that play a key role in modulating the immunological response to implanted biomaterials and other cells, either allogenic or autologous, further increase the complexity [92]. Inhibiting or modulating the immune response could potentially boost cartilage regeneration. However, it is 
unclear whether the immune system has a functional impact on more complex repair processes.

\section{Does the Genome Lead to Variability of Outcomes?}

It is better that early-stage in vivo experiments are initially conducted in smaller animal models. Biocompatibility and bioactivity should be confirmed initially in small animals because of the genetic similarities between animals and humans. In the late-stage in vivo experiments, larger animal models could be used for testing clinical hypothesis and biomechanical quality of implants because of their more similar anatomical features to humans. Additionally, researchers should try to create more experimental systems in animal models because of limitations imposed by cost economics and ethical standards. Further animal studies are still required in tissue engineering for cartilage regeneration with the aim of increasing their predictive value.

\section{Contribution to Cartilage Tissue Engineering}

A total of 1392 titles and abstracts were reviewed preliminarily, in which 976 studies involved in vivo research on cartilage tissue engineering. It was estimated that the number of various species of animals sacrificed was 26,667 . The distribution of studies involving different types of animal models is presented in Fig. 1. The most frequent type of animal sacrificed in basic research is rabbits. With an increase in animal body size, the sample size decreased significantly. Various factors, including ethical reasons, feeding difficulty, and economic costs, may limit the widespread use of larger animals. More importantly, there is an evident trend that the relative contribution of each type of animal model is improved with increasing body size (Fig. 1). We could therefore infer that animals with larger size close to humans may reduce the number of laboratory animals required and lead to the generation of more reliable results. However, for the initial stage of in vivo studies, it is advantageous to utilize small animals to confirm some crucial parameters.

Acknowledgments The authors would like to acknowledge support from the National Basic Research Program of China (973 Program) (2012CB619100) and the National Natural Science Foundation of China (81471800, 81271722, 81501919).

\section{Compliance with Ethical Standards}

Conflict of Interest Dan Xing, Jiaqing Chen, Jiabei Yang, Boon Chin Heng, Zigang Ge, and Jianhao Lin declare that they have no conflict of interest.

Human and Animal Rights and Informed Consent This article does not contain any studies with human subjects performed by any of the authors. With regard to the authors' research cited in this paper, all institutional and national guidelines for the care and use of laboratory animals were followed.

\section{Appendix}

Table 2 Search strategy in PubMed

\begin{tabular}{|c|c|c|}
\hline Search & Query & $\begin{array}{l}\text { Items } \\
\text { found }\end{array}$ \\
\hline \#52 & $\begin{array}{l}\text { Search (((\#44 AND \#38 AND \#35 AND \#27) } \\
\text { AND (“2005/10/01”[PDat] : “2015/10/01” } \\
\text { [PDat]) AND English[lang])) NOT review } \\
\text { [Filter] Filters: Publication date from } \\
\text { 2005/10/01 to 2015/10/01; English }\end{array}$ & 1547 \\
\hline \#47 & $\begin{array}{l}\text { Search (\#44 AND \#38 AND \#35 AND \#27) } \\
\text { Filters: Publication date from 2005/10/01 } \\
\text { to 2015/10/01; English }\end{array}$ & 1790 \\
\hline$\# 44$ & Search (\#39 OR \#41 OR \#42) & 282,199 \\
\hline \#42 & Search scaffold[Title/Abstract] & 36,438 \\
\hline \#41 & Search "tissue engineering"[Title/Abstract] & 24,952 \\
\hline \#39 & Search repair[Title/Abstract] & 234,109 \\
\hline \#38 & Search (\#36 OR \#37) & 331,825 \\
\hline \#37 & Search defects[Title/Abstract] & 208,451 \\
\hline \#36 & Search defect[Title/Abstract] & 163,534 \\
\hline \#35 & $\begin{array}{l}\text { Search (\#28 OR \#29 OR \#30 OR \#31 } \\
\text { OR \#32 OR \#33 OR \#34) }\end{array}$ & 78,468 \\
\hline \#34 & Search subcartilaginous[Title/Abstract] & 4 \\
\hline \#33 & Search "subchondral bone"[Title/Abstract] & 3677 \\
\hline \#32 & Search cartilages[Title/Abstract] & 4114 \\
\hline \#31 & Search subchondral[Title/Abstract] & 5384 \\
\hline \#30 & Search chondral[Title/Abstract] & 2498 \\
\hline \#29 & $\begin{array}{l}\text { Search (chondrocyte[Title/Abstract] OR } \\
\text { chondrocytes[Title/Abstract]) }\end{array}$ & 22,718 \\
\hline \#28 & Search cartilage[Title/Abstract] & 65,663 \\
\hline \#27 & $\begin{array}{l}\text { Search } \# 1 \text { OR \#2 OR \#3 OR \#4 OR \#5 } \\
\text { OR \#6 OR \#7 OR \#8 OR \#9 OR \#10 } \\
\text { OR \#11 OR \#12 OR \#13 OR \#14 } \\
\text { OR \#15 OR \#16 OR \#17 OR \#19 OR \#20) }\end{array}$ & $18,488,859$ \\
\hline$\# 20$ & Search horses[Title/Abstract] & 26,184 \\
\hline \#19 & Search horse[Title/Abstract] & 29,464 \\
\hline \#17 & Search goats[Title/Abstract] & 15,616 \\
\hline \#16 & Search goat[Title/Abstract] & 16,069 \\
\hline \#15 & Search sheep[Title/Abstract] & 79,130 \\
\hline \#14 & Search "in vivo"[Title/Abstract] & 701,544 \\
\hline \#13 & Search mini-pigs[Title/Abstract] & 590 \\
\hline \#12 & Search mini-pig[Title/Abstract] & 288 \\
\hline \#11 & Search rabbits[Title/Abstract] & 115,757 \\
\hline \#10 & Search Rabbit[Title/Abstract] & 163,212 \\
\hline \#9 & Search mice[Title/Abstract] & 699,443 \\
\hline \#8 & Search mouse[Title/Abstract] & 492,817 \\
\hline \#7 & Search pigs[Title/Abstract] & 102,964 \\
\hline \#6 & Search pig[Title/Abstract] & 116,654 \\
\hline \#5 & Search dogs[Title/Abstract] & 144,632 \\
\hline$\# 4$ & Search dog[Title/Abstract] & 80,939 \\
\hline$\# 3$ & Search rat[Title/Abstract] & 782,455 \\
\hline \#2 & Search rats[Title/Abstract] & 740,008 \\
\hline$\# 1$ & Search animal[MeSH Terms] & $18,170,673$ \\
\hline
\end{tabular}




\section{References}

Papers of particular interest, published recently, have been highlighted as:

- Of importance

- Of major importance

1. Acosta $\mathrm{CA}$ et al. Gene expression and proliferation analysis in young, aged, and osteoarthritic sheep chondrocytes effect of growth factor treatment. J Orthop Res. 2006;24(11):2087-94.

2. Zhou Q. Balancing the welfare: the use of non-human primates in research. Trends Genet. 2014;30(11):476-8.

3. Hardingham T, Tew S, Murdoch A. Tissue engineering: chondrocytes and cartilage. Arthritis Res. 2002;4 Suppl 3:S63-8.

4. Ge Z et al. Osteoarthritis and therapy. Arthritis Rheum. 2006;55(3): 493-500.

5. Brown TD et al. Posttraumatic osteoarthritis: a first estimate of incidence, prevalence, and burden of disease. J Orthop Trauma. 2006;20(10):739-44.

6. Huey DJ, Hu JC, Athanasiou KA. Unlike bone, cartilage regeneration remains elusive. Science. 2012;338(6109):917-21.

7. Vaquero J, Forriol F. Knee chondral injuries: clinical treatment strategies and experimental models. Injury. 2012;43(6):694-705.

8. Simon TM, Aberman HM. Cartilage regeneration and repair testing in a surrogate large animal model. Tissue Eng B Rev. 2010;16(1): 65-79.

9. Ahern BJ et al. Preclinical animal models in single site cartilage defect testing: a systematic review. Osteoarthr Cartil. 2009;17(6): 705-13.

10. Chu CR, Szczodry M, Bruno S. Animal models for cartilage regeneration and repair. Tissue Eng B Rev. 2010;16(1):105-15.

$11 . \bullet$ de Vries RB et al. Reducing the number of laboratory animals used in tissue engineering research by restricting the variety of animal models. Articular cartilage tissue engineering as a case study. Tissue Eng B Rev. 2012;18(6):427-35. The authors used cartilage tissue engineering as an example to review the necessity of reducing the number of laboratory animals. Firstly, they gave an overview of the various animal models utilized in cartilage tissue engineering studies. Secondly, they commented on the number of animals used. Finally, they concluded that it is possible to reduce the number of animals utilized by using larger animal models. However, there was a lack of feedback on extrapolation and correlation between preclinical and clinical studies.

12. Plopper CG, Hyde DM. The non-human primate as a model for studying COPD and asthma. Pulm Pharmacol Ther. 2008;21(5): 755-66.

13. Reifenrath $\mathrm{J}$ et al. Replacement, refinement, and reduction: necessity of standardization and computational models for long bone fracture repair in animals. J Biomed Mater Res A. 2014;102(8): 2884-900.

14. Lucken RN. The five Rs: refinement, reduction, replacement. A regulatory revolution. Dev Biol Stand. 1996;86:67-72.

15. U.S. National Institutes of Health. Laboratory animal welfare: Public Health Service policy on humane care and use of laboratory animals by awardee institutions; notice. Fed Regist. 1985;50(90): 19584-5.

16. Nemoto H, Watson D, Masuda K. Transplantation of tissueengineered cartilage in an animal model (xenograft and autograft): construct validation. Methods Mol Biol. 2015;1340:247-59.

17. Collignon $\mathrm{P}$ et al. World Health Organization ranking of antimicrobials according to their importance in human medicine: a critical step for developing risk management strategies for the use of antimicrobials in food production animals. Clin Infect Dis. 2009;49(1): $132-41$.
18. Winthrop Z, Pinkowsky G, Hennrikus W. Surgical treatment for osteochondritis dessicans of the knee. Curr Rev Musculoskelet Med. 2015;8:467-75.

19. Frisbie DD et al. Arthroscopic subchondral bone plate microfracture technique augments healing of large chondral defects in the radial carpal bone and medial femoral condyle of horses. Vet Surg. 1999;28(4):242-55.

20. Tuncay I et al. The effect of hyaluronan combined with microfracture on the treatment of chondral defects: an experimental study in a rabbit model. Eur J Orthop Surg Traumatol. 2013;23(7): $753-8$.

21. Mithoefer K et al. The microfracture technique for the treatment of articular cartilage lesions in the knee. A prospective cohort study. J Bone Joint Surg Am. 2005;87(9):1911-20.

22. Shao $\mathrm{X}$ et al. Repair of large articular osteochondral defects using hybrid scaffolds and bone marrow-derived mesenchymal stem cells in a rabbit model. Tissue Eng. 2006;12(6):1539-51.

23. Shi D et al. Photo-cross-linked scaffold with kartogenin-encapsulated nanoparticles for cartilage regeneration. ACS Nano. 2016;10(1):1292-9

24. Zhang $\mathrm{J}$ et al. Probing cell-matrix interactions in RGD-decorated macroporous poly (ethylene glycol) hydrogels for 3D chondrocyte culture. Biomed Mater. 2015;10(3):035016.

25. Ge $\mathrm{Z}$ et al. Functional biomaterials for cartilage regeneration. J Biomed Mater Res A. 2012;100(9):2526-36.

26. Ladet SG et al. Multi-membrane chitosan hydrogels as chondrocytic cell bioreactors. Biomaterials. 2011;32(23):5354-64.

27. Tang $X$ et al. Evolving concepts of chondrogenic differentiation: history, state-of-the-art and future perspectives. Eur Cell Mater. 2015;30:12-27.

28. Doran PM. Cartilage tissue engineering: what have we learned in practice? Methods Mol Biol. 2015;1340:3-21. The handbook provides careful and detailed protocols for experimental work covering a broad range of objectives for cartilage synthesis and regeneration. It also describes clearly and in detail the key practical skills required in this field.

29. Cortial D et al. Activation by IL-1 of bovine articular chondrocytes in culture within a 3D collagen-based scaffold. An in vitro model to address the effect of compounds with therapeutic potential in osteoarthritis. Osteoarthr Cartil. 2006;14(7):631-40.

30. Ortved KF et al. Implantation of rAAV5-IGF-I transduced autologous chondrocytes improves cartilage repair in full-thickness defects in the equine model. Mol Ther. 2015;23(2):363-73.

31. Lamplot JD et al. Reversibly immortalized mouse articular chondrocytes acquire long-term proliferative capability while retaining chondrogenic phenotype. Cell Transplant. 2015;24(6): 1053-66.

32. Bianchi $\mathrm{G}$ et al. Microenvironment and stem properties of bone marrow-derived mesenchymal cells. Wound Repair Regen. 2001;9(6):460-6.

33. Grassel S, Ahmed N. Influence of cellular microenvironment and paracrine signals on chondrogenic differentiation. Front Biosci. 2007;12:4946-56

34. Wu L, Post JN, Karperien M. Engineering cartilage tissue by pellet coculture of chondrocytes and mesenchymal stromal cells. Methods Mol Biol. 2015;1226:31-41.

35. Vats A et al. Chondrogenic differentiation of human embryonic stem cells: the effect of the micro-environment. Tissue Eng. 2006;12(6):1687-97.

36. Zheng D et al. Controlled chondrogenesis from adipose-derived stem cells by recombinant transforming growth factor-beta3 fusion protein in peptide scaffolds. Acta Biomater. 2015;11:191-203.

37. Haisch A et al. Macroencapsulation of human cartilage implants: pilot study with polyelectrolyte complex membrane encapsulation. Biomaterials. 2000;21(15):1561-6. 
38. Zhu Y et al. The influence of Chm-I knockout on ectopic cartilage regeneration and homeostasis maintenance. Tissue Eng A. 2015;21(3-4):782-92.

39. Fitzgerald $\mathbf{J}$ et al. Evidence for articular cartilage regeneration in MRL/MpJ mice. Osteoarthr Cartil. 2008;16(11):1319-26.

40. Malda $J$ et al. Of mice, men and elephants: the relation between articular cartilage thickness and body mass. PLoS ONE. 2013;8(2): e57683.

41. Matsuoka $\mathrm{M}$ et al. An articular cartilage repair model in common C57B1/6 mice. Tissue Eng C Methods. 2015;21(8):767-72.

42. Sakata R, Iwakura T, Reddi AH. Regeneration of articular cartilage surface: morphogens, cells, and extracellular matrix scaffolds. Tissue Eng B Rev. 2015;21(5):461-73.

43. Lammi PE et al. Strong hyaluronan expression in the full-thickness rat articular cartilage repair tissue. Histochem Cell Biol. 2001;115(4): 301-8.

44. Oshima $\mathrm{Y}$ et al. Fate of transplanted bone-marrow-derived mesenchymal cells during osteochondral repair using transgenic rats to simulate autologous transplantation. Osteoarthr Cartil. 2004;12(10): 811-7.

45. Zwolanek D et al. Betal integrins mediate attachment of mesenchymal stem cells to cartilage lesions. BioRes Open Access. 2015;4(1):39-53.

46. Shimizu $\mathrm{R}$ et al. Repair mechanism of osteochondral defect promoted by bioengineered chondrocyte sheet. Tissue Eng A. 2015;21(5-6):1131-41.

47. Lin YX et al. In vitro and in vivo evaluation of the developed PLGA/HAp/Zein scaffolds for bone-cartilage interface regeneration. Biomed Environ Sci. 2015;28(1):1-12.

48. Ma X et al. Repair of osteochondral defects by mosaicplasty and allogeneic BMSCs transplantation. Int J Clin Exp Med. 2015;8(4): 6053-9.

49. Sermer $\mathrm{C}$ et al. The addition of platelet-rich plasma to scaffolds used for cartilage repair: a review of human and animal studies. Arthroscopy. 2015;31(8):1607-25.

50. Hunziker EB. Biologic repair of articular cartilage. Defect models in experimental animals and matrix requirements. Clin Orthop Relat Res. 1999;(367 Suppl):S135-46.

51. Shao XX et al. Evaluation of a hybrid scaffold/cell construct in repair of high-load-bearing osteochondral defects in rabbits. Biomaterials. 2006;27(7):1071-80.

52. Hunziker EB. Articular cartilage repair: basic science and clinical progress. A review of the current status and prospects. Osteoarthr Cartil. 2002;10(6):432-63.

53. Deng $\mathrm{S}$ et al. Miscellaneous animal models accelerate the application of mesenchymal stem cells for cartilage regeneration. Curr Stem Cell Res Ther. 2014;9(3):223-33.

54. Feczko $\mathrm{P}$ et al. Experimental results of donor site filling for autologous osteochondral mosaicplasty. Arthroscopy. 2003;19(7):75561.

55. Sosio $\mathrm{C}$ et al. Osteochondral repair by a novel interconnecting collagen-hydroxyapatite substitute: a large-animal study. Tissue Eng A. 2015;21(3-4):704-15.

56. Frisbie DD, Cross MW, Mcllwraith CW. A comparative study of articular cartilage thickness in the stifle of animal species used in human pre-clinical studies compared to articular cartilage thickness in the human knee. Vet Comp Orthop Traumatol. 2006;19(3):142-6.

57. Ho ST et al. The evaluation of a biphasic osteochondral implant coupled with an electrospun membrane in a large animal model. Tissue Eng A. 2010;16(4):1123-41.

58. Tibesku $\mathrm{CO}$ et al. Hyaline cartilage degenerates after autologous osteochondral transplantation. J Orthop Res. 2004;22(6):1210-4.

59. Siebert $\mathrm{CH}$ et al. Healing of osteochondral grafts in an ovine model under the influence of bFGF. Arthroscopy. 2003;19(2):182-7.
60. Wang $\mathrm{L}$ et al. Multiparametric MRI of epiphyseal cartilage necrosis (osteochondrosis) with histological validation in a goat model. PLoS ONE. 2015;10(10): 0140400.

61. Kon E et al. Osteochondral regeneration with a novel aragonitehyaluronate biphasic scaffold: up to 12-month follow-up study in a goat model. J Orthop Surg Res. 2015;10:81.

62. Pei $\mathrm{Y}$ et al. Repairing the osteochondral defect in goat with the tissue-engineered osteochondral graft preconstructed in a doublechamber stirring bioreactor. Biomed Res Int. 2014;2014:219203.

63. Hurtig MB et al. Effects of lesion size and location on equine articular cartilage repair. Can J Vet Res. 1988;52(1):137-46.

64. Edwards 3rd RB et al. Comparison of mechanical debridement and radiofrequency energy for chondroplasty in an in vivo equine model of partial thickness cartilage injury. Osteoarthr Cartil. 2007;15(2): 169-78.

65. Huckle J et al. Differentiated chondrocytes for cartilage tissue engineering. Novartis Found Symp. 2003;249:103-12. discussion 112-7, 170-4, 239-41.

66. Sesia SB et al. Anti-inflammatory/tissue repair macrophages enhance the cartilage-forming capacity of human bone marrowderived mesenchymal stromal cells. J Cell Physiol. 2015;230(6): 1258-69.

67. Rederstorff E et al. Enriching a cellulose hydrogel with a biologically active marine exopolysaccharide for cell-based cartilage engineering. J Tissue Eng Regen Med. 2015.

68. Mehrabani D et al. The healing effect of adipose-derived mesenchymal stem cells in full-thickness femoral articular cartilage defects of rabbit. Int J Organ Transplant Med. 2015;6(4):165-75.

69. Dearmin MG et al. Chondroprotective effects of zoledronic acid on articular cartilage in dogs with experimentally induced osteoarthritis. Am J Vet Res. 2014;75(4):329-37.

70. Choate CJ et al. Effect of lateral meniscectomy and osteochondral grafting of a lateral femoral condylar defect on contact mechanics: a cadaveric study in dogs. BMC Vet Res. 2013;9:53.

71. Fitzpatrick $\mathrm{N}$ et al. Osteochondral autograft transfer for the treatment of osteochondritis dissecans of the medial femoral condyle in dogs. Vet Comp Orthop Traumatol. 2012;25(2):135-43.

72. Hjelle $\mathrm{K}$ et al. Articular cartilage defects in 1,000 knee arthroscopies. Arthroscopy. 2002;18(7):730-4.

73. Nakamura T et al. Arthroscopic, histological and MRI analyses of cartilage repair after a minimally invasive method of transplantation of allogeneic synovial mesenchymal stromal cells into cartilage defects in pigs. Cytotherapy. 2012;14(3):327-38.

74. Ortved KF, Nixon AJ. Cell-based cartilage repair strategies in the horse. Vet J. 2015.

75. Gratz KR et al. Biomechanical assessment of tissue retrieved after in vivo cartilage defect repair: tensile modulus of repair tissue and integration with host cartilage. J Biomech. 2006;39(1):138-46.

76. Wei X, Gao J, Messner K. Maturation-dependent repair of untreated osteochondral defects in the rabbit knee joint. J Biomed Mater Res. 1997;34(1):63-72.

77. Andronescu AA et al. Associations between early radiographic and computed tomographic measures and canine hip joint osteoarthritis at maturity. Am J Vet Res. 2015;76(1):19-27. The authors reviewed some minimally invasive, quantitative, and real-time techniques; bioluminescent imaging systems; histological scoring systems; and other novel techniques for evaluating in vivo tissue engineering studies. It was concluded that there is a need for improved techniques to evaluate implanted biomaterials within the host.

78. Trachtenberg JE, Vo TN, Mikos AG. Pre-clinical characterization of tissue engineering constructs for bone and cartilage regeneration. Ann Biomed Eng. 2015;43(3):681-96.

79. Kiviranta $\mathrm{P}$ et al. Indentation diagnostics of cartilage degeneration. Osteoarthr Cartil. 2008;16(7):796-804. 
80. Saarakkala S et al. Quantitative ultrasound imaging detects degenerative changes in articular cartilage surface and subchondral bone. Phys Med Biol. 2006;51(20):5333-46.

81. Palmer AW, Guldberg RE, Levenston ME. Analysis of cartilage matrix fixed charge density and three-dimensional morphology via contrast-enhanced microcomputed tomography. Proc Natl Acad Sci U S A. 2006;103(51):19255-60.

82. Yang Q et al. A cartilage ECM-derived 3-D porous acellular matrix scaffold for in vivo cartilage tissue engineering with PKH26labeled chondrogenic bone marrow-derived mesenchymal stem cells. Biomaterials. 2008;29(15):2378-87.

83. Ramaswamy $\mathrm{S}$ et al. Magnetic resonance imaging of chondrocytes labeled with superparamagnetic iron oxide nanoparticles in tissueengineered cartilage. Tissue Eng A. 2009;15(12):3899-910.

84. Nieminen $\mathrm{HJ}$ et al. Determining collagen distribution in articular cartilage using contrast-enhanced micro-computed tomography. Osteoarthr Cartil. 2015;23(9):1613-21.

85. Williams GM, Klisch SM, Sah RL. Bioengineering cartilage growth, maturation, and form. Pediatr Res. 2008;63(5):527-34
86. Peck $\mathrm{Y}$ et al. A preclinical evaluation of an autologous living hyaline-like cartilaginous graft for articular cartilage repair: a pilot study. Sci Rep. 2015;5:16225.

87. Wu JP, Kirk TB, Zheng MH. Study of the collagen structure in the superficial zone and physiological state of articular cartilage using a 3D confocal imaging technique. J Orthop Surg Res. 2008;3:29.

88. Kasemkijwattana $\mathrm{C}$ et al. Autologous bone marrow mesenchymal stem cells implantation for cartilage defects: two cases report. J Med Assoc Thail. 2011;94(3):395-400.

89. Enea D et al. One-step cartilage repair in the knee: collagen-covered microfracture and autologous bone marrow concentrate. A pilot study. Knee. 2015;22(1):30-5.

90. Gobbi A et al. One-step cartilage repair with bone marrow aspirate concentrated cells and collagen matrix in full-thickness knee cartilage lesions: results at 2-year follow-up. Cartilage. 2011;2(3):286-99.

91. Pastides $\mathrm{P}$ et al. Stem cell therapy for human cartilage defects: a systematic review. Osteoarthr Cartil. 2013;21(5):646-54.

92. Eming SA et al. Interrelation of immunity and tissue repair or regeneration. Semin Cell Dev Biol. 2009;20(5):517-27. 